\title{
Chronic Fistula After Revision Laparoscopic Sleeve Gastrectomy
}

\author{
Giuseppe S. Sica, MD, PhD, Marco D'Eletto, MD, Cristina Fiorani, MD, Andrea Divizia, MD, \\ Paolo Gentileschi, MD, Domenico Benavoli, MD, Manfredi Tesauro, MD, PhD, \\ Achille L. Gaspari, MD \\ Department of General Surgery, Tor Vergata University of Rome, Rome, Italy (all authors).
}

\begin{abstract}
Laparoscopic sleeve gastrectomy (LSG) is a safe and effective bariatric surgery procedure. Leaks along the staple line are serious complications of the procedure and can result in significant morbidity. Treatment depends on the timing, site, and clinical consequence of the leak. We describe the case of a young, formerly obese woman who presented with a chronic gastric fistula at the esophagogastric junction after an LSG. Treatment of this complication required multiple interventions by a highly specialized team. Physicians' decision-making was difficult throughout the entire process, and complete healing of the fistula was accomplished 20 months after the LSG. A multidisciplinary approach is mandatory in the treatment of a chronic fistula from LSG, but there is no standard treatment strategy.
\end{abstract}

Key Words: Morbid obesity, Laparoscopy, Sleeve gastrectomy.

Citation Sica GS, D'Eletto M, Fiorani C, Divizia A, Gentileschi P, Benavoli D, Tesauro M, Gaspari AL. Chronic fistula after revision laparoscopic sleeve gastrectomy. CRSLS e2014.00108. DOI: 10.4293/CRSLS.2014.00108.

Copyright $\odot 2014$ SLS This is an open-access article distributed under the terms of the Creative Commons Attribution-Noncommercial-ShareAlike 3.0 Unported license, which permits unrestricted noncommercial use, distribution, and reproduction in any medium, provided the original author and source are credited.

Address correspondence to: Cristina Fiorani, Tor Vergata University of Rome, General Surgery, viale Oxford 8100133 Rome, Italy. Telephone: (+39) 062-090-2976, E-mail: fiorani.cristina@gmail.com

\section{INTRODUCTION}

Laparoscopic sleeve gastrectomy (LSG) is a beneficial operation to aid weight loss in morbidly obese patients. Currently, LSG is a consolidated surgical procedure and, in general, bariatric surgeons considered it to be not only safe and effective but also somewhat simpler than other procedures requiring anastomoses, such as the gastric bypass. However, there are several technical differences to fashioning the gastric tubule. Various bougie sizes are used; some surgeons use buttress material to reinforce the staple line, some oversew it wholly or in part, and some do nothing.

Dehiscence along the stapled line occurs in as many as $5.5 \%$ of LSGs,${ }^{1-3}$ and these leaks usually lead to significant morbidity. ${ }^{4}$ Furthermore, leaks from LSG seem to occur more often when the procedure is performed on patients who have already undergone a gastric band procedure. The management is strictly dependent on the time from the leak, but most physicians recommend a conservative treatment or an endoscopic salvage procedure. We present the case report of a young formerly obese patient with a chronic esophagogastric junction (EGJ) fistula after revision LSG.

\section{CASE REPORT}

A 35-year-old woman was referred to our unit because of a chronic fistula after LSG. Her medical history included a laparoscopic gastric banding implanted at her local hospital to manage morbid obesity (body mass index [BMI] 40). Two years after the band surgery, she underwent an LSG at the same center because she had regained much of the weight she had lost (BMI: 38). There was no record in her medical notes regarding the size of the bougie used for sizing the resection. She had an apparently uneventful recovery and was sent home after 5 days as is common practice in a district hospital of this country in some regions with difficult logistics. Fifteen days after her surgery, she was readmitted to the same hospital because of fever and abdominal pain. A computed tomography (CT) scan of her abdomen showed a large fluid collection localized in the left subphrenic space and a smaller one located in the mediastinum, along the esophagus. She was 


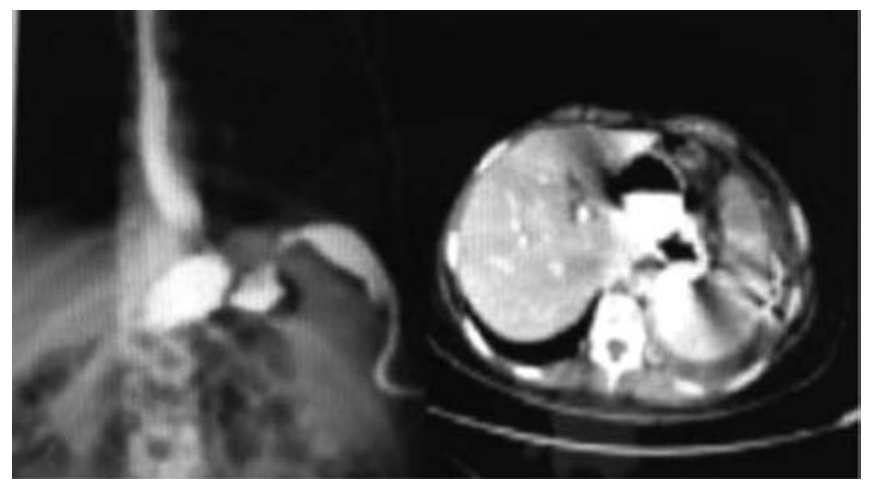

Figure 1. A, Oral contrast post LSG; B, Computed tomography scan showing subphrenic collection.

taken to the operating room and the collections were drained through a left subcostal incision. Three drains were left in place and eventually removed before the patient was sent home on a soft diet, 30 days after her second admission. A CT scan with oral contrast performed before she was discharged showed normal findings. Five weeks later, the patient presented again to the hospital because of abdominal pain and fever. A CT scan again showed a large fluid collection in the left subphrenic space. A percutaneous drain was inserted, and the abscess was drained. The patient was sent home on a liquid diet, receiving total parenteral nutrition, and with a pig-tail drain in place. Approximately $60 \mathrm{~mL}$ of foul mucoid fluid drained daily from the controlled fistula at the time. Two months later, the patient self-referred to our unit. On admission, she was still overweight (BMI 36) and in poor general condition, with a pulse rate of $120 \mathrm{bpm}$ but normal blood pressure. The abdomen was soft and the surgical scars were well-healed. Laboratory blood analysis results showed anemia, a low white cell count, and an increase in C-reactive protein. High levels of amylase were found in the fluid draining from the abdomen, suggesting an open fistula with persistent leak from the stomach and not a simple residual abscess from previous surgery. Radiographs taken with hydrosoluble oral contrast showed the presence of a leak at the EGJ, and a CT scan revealed fluid collection next to the leak, with air bubbles (Figure 1 [A\&B] ). A first attempt to close the internal orifice was done using endoscopic clips and a covered self-expandable stent; the methylene blue test showed a persistent leak 2 days after the procedure, and the stent had to be endoscopically retrieved because it had migrated to the distal stomach and across the duodenum. At that point, under the skin of her left subcostal incision, a lump and some redness in accordance with a superficial abscess (gastrocutaneous fistula) was seen. The patient was taken back to operating room and the previous scar was reopened. Pus was drained from the subcutaneous space and the fistulous tract followed down into the abdomen. Tenacious bowel adhesions had to be freed to access the abdominal cavity; a severe inflammatory reaction was present, most prominently in the epigastric region and upper left abdominal quadrant. The anterior wall of the stomach was undetachable from the left lobe of the liver, and the spleen was stuck to the upper left subdiaphragmatic space. Eventually, after drainage of the abscess deep into the abdominal cavity, it was possible to reach the diaphragmatic crura, dissect the inferior esophagus, and localize a $1-\mathrm{cm}$ hole at the EGJ. In that area the stomach appeared thickened and inflamed, and it was not possible to determine whether the plication from the previous band was completely reduced. A large hole eventually became visible at the OGJ. After a wide excision of the lower esophagus, Hiss angle, and part of the gastric fundus, direct repair with buttress sutures was attempted. Suturing a high sleeve leak or any other chronic hole in the intestine does not work. However, when the leaking hole was reached, we decided to resect the inflamed fibrotic tissue to obtain well-vascularized tissue to stitch together. This was done in hopes that it would have healed but also to create a somewhat younger leak to treat, in case of failure. Postoperative recovery was uneventful, and the patient was again allowed a soft diet after contrast swallow test results were negative. She was discharged home nine days after surgery and did well for a while. Two months later, she had to be readmitted because of abdominal pain and fever. A subcutaneous abscess was palpable under the abdominal scar and was surgically drained. A smaller leakage of oral contrast was again visible on a radiograph. At this point, a covered, self-expandable, and retrievable stent was placed endoscopically and the patient was soon sent home on a soft diet. The stent was removed after six weeks, and the swallow test with water-soluble contrast showed no persistent leakage. Three months later, a new subcutaneous abscess spontaneously draining pus revealed a persistent gastrocutaneous fistula. The patient underwent an endoscopic placement of a new covered, self-expandable, and retrievable stent (Figure 2) (TaeWoong Niti-S Esophageal stent Mega, TaeWoong Medical, Gyeonggi-do, South Korea). At the same time, a nasojejunal (NJ) tube was inserted for enteral feeding and kept in place for six weeks. Both the stent and NJ tube were removed after six weeks, and the post-stent removal contrast swallow results were negative for leakage and showed good emptying. Two weeks later, and 14 months after her first admission to our unit, she had to be readmitted with the same persistent 


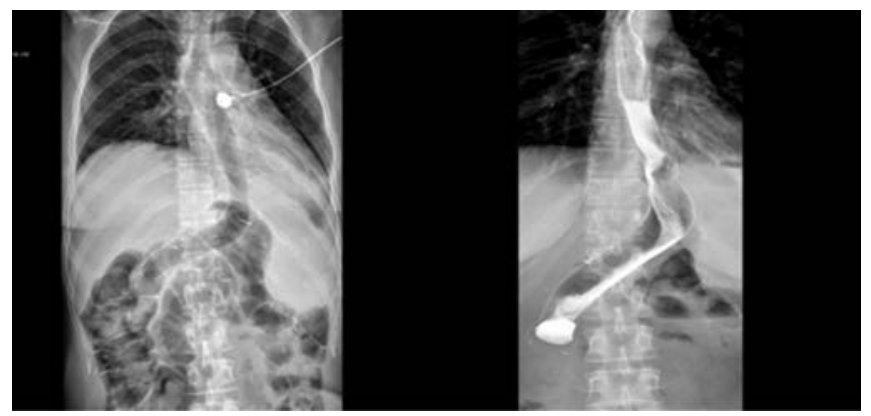

Figure 2. The TaeWoong Niti-S Esophageal stent Mega in place.

problem. The new subdiaphragmatic abscess was drained and enteral nutrition through a NJ tube was started. The patient refused the proposed gastric resection and interposition of jejunum (Merendino operation) and was kept on enteral feeding for another few months. Her fistula eventually healed, and she is now doing well almost two years after her LSG.

\section{DISCUSSION}

LSG is an effective procedure for the management of obesity. Historically, it represented the first stage of a two-stage bypass operation in high-risk patients, but it was soon considered a definitive bariatric operation itself. The favor LSG has gained among bariatric surgeons lies in the fact that it is less technically challenging than Rouxen-Y gastric bypass and does not require implantation of foreign material, and weight loss is achieved by both restricted food intake and hormonal modulation. Furthermore, LSG is an overall safe procedure with an acceptable rate of complications, although failure of the staple line is a serious problem that can occur in $0.5 \%$ and $5 \%$ of patients according to various studies. ${ }^{5-7}$ Clinical presentations of leaks vary from asymptomatic patients, who have their leaks detected during the routine postoperative contrast study, to patients presenting with symptoms of sepsis and multiorgan failure. ${ }^{5}$ Gastric leaks after LSG can be classified according to the time of occurrence, radiological findings, clinical behavior, and cause of the leak. ${ }^{8-10}$ An early leak usually presents within the third postoperative day, whereas a late leak should be seen after the eighth postoperative day. Based on upper gastrointestinal contrast study, or in accordance with CT scan findings, leaks can be often correlated to their clinical presentation: type I or subclinical leak, and type II or clinical leak. ${ }^{11,12}$

Early symptomatic leaks could possibly be managed by laparoscopic suture, whereas treatment of intermediate or late leaks depends on patient condition and clinical man- ifestations of the fistula. Although some cases can be managed conservatively, persistent fistulas require an invasive approach. Endoscopic clips, injection of sealants, and stent placement are less invasive modalities that can be attempted to avoid subsequent surgery.

This is an interesting case report that highlights what can go wrong with a sleeve gastrectomy. Because there is a misconception that sleeve gastrectomies are somehow safer than gastric bypass, it is important to remind surgeons and patients the complications that can occur with them.

Furthermore, the case report is of a young woman who had a persistent leak after a revision procedure for morbid obesity. Initially, she had laparoscopic gastric band surgery, followed by a sleeve gastrectomy because of weight gain. An LSG was done in the same operation when the band was removed, but unfortunately her sleeve leaked and she required intensive treatments for almost two years.

This case is representative of a possible cause of LSG leak, particularly after revision surgery. Unfortunately, there is no record of whether-when the band was removed-the surgeons adequately opened the gastrogastric plication to ensure that the new staple line was in fact forming on two fully released, unencapsulated, and flat surfaces of stomach.

After LSB, it is possible to observe a deformity of the stomach, and if the stomach is not completely flattened, the remaining deformity may result in malposition of the gastric fundic wall or, worse, more than two gastric walls can be included in the staple line. Apart from this consideration, revision LSG is considered to carry a higher risk of complications because the gastric resection is undertaken upon scarred, encapsulated, thickened gastric tissue. The debate of whether the LSG should be performed at the same time as the band removal is still open. ${ }^{16}$ However, most authors agree on a single-stage procedure. ${ }^{17,18}$

In this case, the fistula was again localized at the EGJ. The patient was a young, obese, and otherwise healthy individual (eg, no diabetes), and the leak was a late leak. She was taken back for surgery and a large subcostal incision was made to gain access to the abdomen to drain the abscess. Considerations need to be made as follows: in case of a late leak, it may be better to drain the collection, rest the bowel, and stent the leak. It is also possible to reoperate and redo laparoscopy, washing the abdominal cavity, with no attempt to suture the hole. Open surgery 
on morbidly obese patients in this setting can lead to disastrous consequences.

Thirty days after the operation, and with the assumption that the fistula had healed because of a negative contrast swallow, the patient was sent home on a soft diet. Unfortunately the fistula reopened or, most likely, it had never completely healed. At this point, a pig-tail drain was inserted and total parenteral nutrition commenced. It is sensible to believe that too little time had passed after the operation to allow complete healing and, as often happens, a negative contrast study can be false. Thus far, no attempt to close the orifice had been made and when she presented to us again, a combined endoscopic procedure was chosen: a self-expanding covered stent was placed over a specially designed clip used to close the internal orifice at the same time. Both devices failed to achieve the goal: in the case of clip placement, the cause of failure became clear at the time of subsequent surgery, where fibrotic and inflamed thick tissue was found at the fistulous orifice; the stent was not appropriately placed and slipped into the stomach. Endo-clipping at the EJG is difficult because of the tangential position of the orifice with respect to the endoscope; however, the main problem is the inflamed tissue, with friable mucosa and fibrotic tissue on the borders. Some authors have recommended mucosal ablation before clipping. It is our belief that clipping a chronic fistula is not an efficacious system to guarantee sealing. Most authors have supported the use of flexible, coated stents as a second step ${ }^{16-20}$; in this way, a temporary bypass over the internal orifice is created and oral feeding can be started. It also allows, when the clinical situation is appropriate, that the patient can temporarily return home. ${ }^{13}$ Any abscess or intraabdominal collection should be drained before placing the stent. The placement of the stent is done under endoscopic or fluoroscopic guidance in accordance with local expertise. Stent migration occurs in as much as one-third of patients, ${ }^{14,15}$ and this is because the prostheses currently employed are mainly designed to be used in esophageal disease with stenosis. In our case, the first stent lasted only a few days, and the second remained in place long term. Unfortunately, possibly giving the shape of the gastric tubule and subsequent increment of intragastric pressure, the fistula relapse and a new stent were deployed. The megastent was chosen in the hopes of also bypassing the right angle at the level of the gastric antrum, which was possibly responsible for the difficult emptying of the stomach. The increase of the intragastric pressure after the sleeve is a known mechanism of problems and possible leaks. This young woman's fistula leaked again two months after reparative surgery. It is unlikely that this was a persistent leak, given the timing and clinical setting, and we therefore hypothesize that the relapse was caused by imperfect emptying. Furthermore, her fistula reopened another two times after having had two subsequent stent placements, and this may indicate that in addition to the ischemic fundus, there was also an imperfect gastric tubule with enhanced intragastric pressure. The last stent was left in place for 6 weeks but, again, the fistula reopened. At this point, redo surgery was discussed with the patient. The idea was to resect the upper part of the stomach and the inferior esophagus to obtain good tissue on both sides and to pull up a limb of jejunum to interpose. The Merendino procedure was chosen rather than a Roux-en-Y gastric bypass, because it allows gastric and duodenal food passage, and in selected cases, such as the one in this discussion, it offers the advantage of maintaining a more physiological status. However, the patient refused the proposed operation after receiving the information. Again she was receiving enteral feeding through a naso-enteral tube, for two months. After this, her fistula finally healed, and she is well 12 months since her last admission and more than two years after her initial presentation. The treatment has been difficult, multidisciplinary, time-consuming, and expensive.

\section{CONCLUSIONS}

Low incidences of leak after LSG have been reported. Leaks after revision surgery are possibly even more difficult to treat because they occur upon scarred, encapsulated, and thickened gastric tissue. There is no unequivocal treatment plan strategy, but stenting of the internal orifice seems to be the most promising method of cure if it is applied with correct timing. Otherwise, bowel rest, adequate drainage, and patience are probably the most effective treatment of chronic gastric fistula. Surgery is always complex and, in case of late leaks, it seems to offer little more than a good drainage of the abdomen.

\section{References:}

1. De Aretxabala X, Leon J, Wiedmaier G, et al. Gastric leak after sleeve gastrectomy: analysis of its management. Obes Surg. 2011;21(8):1232-1237.

2. Clinical Issues: Committee of American Society for Metabolic and Bariatric Surgery. Updated position statement on sleeve gastrectomy as a bariatric procedure. Surg Obes Relat Dis. 2010; 6:1-5.

3. Tan J, Kariyawasam S, Wijeratne T, et al. Diagnosis and management of gastric leaks, after laparoscopic sleeve gastrectomy for morbid obesity. Obes Surg. 2010;20:203-209. 
4. Gagner M. Leaks after sleeve gastrectomy are associated with smaller bougies: prevention and treatment strategies. Surg Laparosc Endosc Percutan Tech. 2010;20(3):166-169.

5. Burgos AM, Braghetto I, Csendes A, et al. Gastric leak after laparoscopic-sleeve gastrectomy for obesity. Obes Surg. 2009;19: 1672-1677.

6. Casella G, Soricelli E, Rizello M, et al. Nonsurgical treatment of staple line leaks after laparoscopic sleeve gastrectomy. Obes Surg. 2009;19:821-826.

7. Stroh C, Birk D, Flade-Kuthe R, et al. Results of sleeve gastrectomy-data from a nationwide survey on bariatric surgery in Germany. Obes Surg. 2009;19(5):632-640.

8. Marquez MF, Ayza MF, Lozano RB, et al. Gastric leak after laparoscopic sleeve gastrectomy. Obes Surg. 2010 Sep; 20(9): 1306-11. doi: 10.1007/s11695-010-0219-7.

9. Bruce J, Krukowski ZH, Al-Khairy G, et al. Systematic review of the definition and measurement of anastomotic leak after gastrointestinal surgery. Br J Surg. 2001 Sep;88(9):1157-68.

10. Baker RS, Foote J, Kemmeter P, et al. The science of stapling and leaks. Obes Surg. 2004 Nov-Dec;14(10):1290-8.

11. Sarkhosh K, Birch DW, Sharma A, Karmali S. Complications associated with laparoscopic sleeve gastrectomy for morbid obesity: a surgeon's guide. Can J Surg. 2013 Oct;56(5):347-52.

12. Nedelcu M, Skalli M, Delhom E, et al. New CT scan classification of leak after sleeve gastrectomy. Obes Surg. 2013 Aug; 23(8):1341-3. doi: 10.1007/s11695-013-1002-3.
13. Berende CAS, De Zoete JP, Nienhuijs SW. Laparoscopic sleeve gastrectomy feasible for bariatric revision surgery. Obes Surg. 2012;22:330-334.

14. Khan OA, Mansour S, Irukulla S, et al. Sleeve gastrectomy for gastric band failures-a prospective study. Int J Surg. 2013;11(5): 407-409.

15. Utech M, Shaheen H, Halter J, et al. Sleeve gastrectomy as a revision procedure for failed gastric banding. Zentralbl Chir. 2014;139(1):79-82.

16. Casella G, Soricelli E, Rizello M, et al. Nonsurgical treatment of staple line leaks after laparoscopic sleeve gastrectomy. Obes Surg. 2009;19:821-826.

17. Serra C, Baltasar A, Andreo L, et al. Treatment of gastric leaks with coated self-expanding stents after sleeve gastrectomy. Obes Surg. 2007;17:866-872.

18. Fukumoto R, Orlina J, McGinty J, et al. Use of polyflex stents in treatment of acute esophageal and gastric leaks after bariatric surgery. Surg Obes Relat Dis. 2006;2:570-572.

19. Eubanks S, Edwards CA, Fearing NM, et al. Use of endoscopic stents to treat anastomotic complications after bariatric surgery. J Am Coll Surg. 2008;206(5):935-938.

20. Salinas A, Baptista A, Santiago E, et al. Self-expandable metal stents to treat gastric leaks. Surg Obes Relat Dis. 2006;2(5):570572. 